\title{
The Effect of Rate Control on Speech Rate and Intelligibility of Dysarthric Speech
}

\author{
Gwen Van Nuffelen ${ }^{\mathrm{a}}$ Marc De Bodt ${ }^{\mathrm{b}}$ Floris Wuyts ${ }^{\mathrm{a}}$ Paul Van de Heyning ${ }^{\mathrm{a}}$ \\ a Department of Oto-Rhino-Laryngology, Head and Neck Surgery, Antwerp University Hospital, Antwerp, and \\ ${ }^{\mathrm{b}}$ Ghent University, Ghent, Belgium
}

\section{Key Words}

Rate control · Intelligibility · Dysarthria

\begin{abstract}
Purpose: This study investigated the effect of rate control methods (RCMs) on speaking rate (SR), articulation rate (AR), and intelligibility in dysarthric speakers. Method: Nineteen dysarthric patients (7 unilateral upper motor neuron dysarthria, 6 hypokinetic, 3 flaccid, 3 ataxic) participated. SR, AR and intelligibility ratings were determined on the basis of 1min recorded reading passages. Seven RCMs were applied: voluntary rate control, hand tapping, alphabet board, pacing board and delayed auditory feedback with a delay of 50 , 100 and 150 ms. Results: Almost all methods resulted in lower mean SRs and ARs $(p<0.05)$. Rate control did not improve overall intelligibility of the dysarthric population. However, a meaningful increase of intelligibility was found in 5 participants. This study indicates that the effect of rate control on intelligibility is independent of habitual speech rate and type of dysarthria. Degree of intelligibility may be an influencing factor. The most effective methods are: voluntary rate control, alphabet board, hand tapping and pacing board. Conclusion: RCMs do result in lower speech rates. Some dysarthric individuals do benefit from one or more RCMs, but rate control may also have an inverse effect on intelligibility.

Copyright $\odot 2009$ S. Karger AG, Basel
\end{abstract}

The purpose of this study was to investigate the effect of several rate control methods (RCMs) on speech rate (speaking rate, SR, and articulation rate, $\mathrm{AR}$ ) and intelligibility in dysarthric speakers. Improving intelligibility is often the primary goal of speech therapy in patients with dysarthria. Speech rate has been considered as the most modifiable variable for improving intelligibility of dysarthric speech $[1,2]$. It has been shown that dysarthric speakers tend to speak near the upper limit of their SR [3, 4]. Reduced rate may lead to increased articulatory displacements, resulting in phonetic events that are more acoustically distinct [5]. Several studies have shown that there is a correlation between speech rate and articulatory precision. In patients with dysarthria secondary to Parkinson's disease (PD), labial movements were judged to be normal at slower SRs, whereas labial movements became hypokinetic as SR increased to the habitual rate [6]. The group findings of a study on amyotrophic lateral sclerosis patients suggest an expansion of the vowel acoustic working space and higher acoustic-perceptual distinctiveness as rate is slowed [7]. In addition, some rate reduction methods enable the patient to produce more appropriate breath group units, which may result in appropriate syntactic units [8]. Next to the impact on speech production, reduction of speech rate may also provide the listeners more time to decode the distorted speech signal [9]. These results suggest that rate control might have a positive effect on the intelligibility of dysarthric speech.

Gwen Van Nuffelen

Department of ORL, Antwerp University Hospital

Bankwegelhof 81, BE-9120 Beveren (Belgium)

Tel. +32382134 85, Fax +32 38214451

E-Mail gwen.van.nuffelen@telenet.be,gwen.van.nuffelen@uza.be 
Table 1. Subjects' characteristics, habitual SR and AR

\begin{tabular}{cllllll}
\hline $\begin{array}{l}\text { Pa- } \\
\text { tient }\end{array}$ & Gender & $\begin{array}{l}\text { Age } \\
\text { years }\end{array}$ & Etiology & Subtype & $\begin{array}{l}\text { Habitual } \\
\text { SR, syl- } \\
\text { lables/s }\end{array}$ & $\begin{array}{l}\text { Habitual } \\
\text { AR, syl- } \\
\text { lables/s }\end{array}$ \\
\hline 1 & male & 24 & MD & flaccid & 2.48 & 3.73 \\
2 & male & 70 & PD & hypokinetic & 3.00 & 5.23 \\
3 & female & 53 & CVA & UUMND & 2.93 & 5.42 \\
4 & female & 17 & trauma & UUMND & 3.52 & 5.54 \\
5 & female & 34 & trauma & flaccid & $0.93^{\mathrm{a}}$ & $2.51^{\mathrm{a}}$ \\
6 & male & 54 & CVA & ataxic & 3.27 & 4.24 \\
7 & male & 44 & CVA & ataxic & 2.92 & $6.51^{\mathrm{b}}$ \\
8 & male & 58 & CVA & hypokinetic & 2.73 & 5.86 \\
9 & male & 61 & PD & hypokinetic & 3.00 & $8.96^{\mathrm{b}}$ \\
10 & male & 37 & MD & flaccid & 2.75 & 4.58 \\
11 & female & 52 & CVA & ataxic & $1.42^{\mathrm{a}}$ & $2.02^{\mathrm{a}}$ \\
12 & male & 57 & CVA & UUMND & 3.10 & 4.53 \\
13 & male & 72 & CVA & UUMND & 3.47 & 4.99 \\
14 & female & 68 & CVA & UUMND & 3.02 & 3.83 \\
15 & male & 72 & CVA & UUMND & 2.62 & 3.68 \\
16 & female & 78 & CVA & UUMND & 2.30 & $3.49^{\mathrm{a}}$ \\
17 & male & 88 & PD & hypokinetic & 2.88 & 4.37 \\
18 & male & 78 & PD & hypokinetic & 3.43 & $6.14^{\mathrm{b}}$ \\
19 & male & 86 & PD & hypokinetic & 2.43 & 5.32 \\
\hline
\end{tabular}

$\mathrm{MD}=$ Muscular dystrophy; CVA = cerebral vascular accident; UUMND = unilateral upper motor neuron dysarthria.

a Speaking rate or articulation rate lower than the lower boundary of the $95 \%$ confidence interval of the control group.

${ }^{b}$ Speaking rate or articulation rate exceeds the upper boundary of the $95 \%$ confidence interval of the control group.

The effect of rate control on intelligibility has been scientifically investigated either by using therapeutic RCMs or by synthetically modifying temporal aspects of recorded speech samples. Hammen et al. [9] examined the effect of three synthetic alterations (pause altered, speech duration altered and pause and speech duration altered) on the intelligibility of dysarthric speech secondary to PD. None of the alterations were found to be more intelligible than samples produced at habitual SRs. This was confirmed in a study by Dagenais et al. [10], in which intelligibility of dysarthric speech remained unchanged across digitally produced rate changes. Flexible or voluntary RCMs (speaking slower on demand) seem to have a quite varied effect on intelligibility. In one study, higher intelligibility estimates following voluntary reduced rates were found in 4 of the 9 patients with amyotrophic lateral sclerosis [7]. Another study revealed no effect of SR adjustments by means of instruction on scaled intelligibility in persons with amyotrophic lateral sclerosis [11]. Also, in the case of patients with multiple sclerosis and PD, voluntary articu- latory rate reduction did not result in significantly higher intelligibility estimates [5]. Contrary to flexible RCMs, positive results were found for rigid methods. Measured sentence intelligibility of both ataxic and hypokinetic dysarthric speakers was found to increase significantly as SR was reduced using pacing methods $[9,12]$. The largest improvement was found when SR was reduced to $60 \%$ of the habitual rate. Delayed auditory feedback has been found to make a positive contribution to the intelligibility of some patients with PD $[8,13,14]$.

It may be concluded that the currently published data concerning the effect of RCMs on intelligibility are rather heterogeneous. In addition, the literature does not reveal any indication of which RCMs are most effective. More research is needed to clarify the value of rate control therapy in dysarthric patients. This study addresses four research questions: (1) Does rate control result in reduced SR and/or reduced AR in dysarthric speakers? (2) Which RCMs have the highest impact on SR and AR? (3) Does rate control result in increased intelligibility of dysarthric speech? (4) Does the effect of rate control on intelligibility depend on the speaker's habitual speech rate, type of dysarthria, selected RCM and/or degree of intelligibility? The aims of this study are to contribute to the knowledge on variables determining the effect of rate control in dysarthria and, secondly, to select the most appropriate and effective RCM.

\section{Method}

\section{Participants}

Nineteen dysarthric speakers, 13 men and 6 women, between 17 and 88 years old (mean age: 58 years) volunteered to participate in this study. All participants reported adequate language, hearing and visual abilities. The etiology of dysarthria varied among the dysarthric speakers. Detailed information per patient concerning age, gender, type, etiology of dysarthria, habitual SR and habitual AR is presented in table 1.

SRs and ARs of the dysarthric speakers were compared with the $95 \%$ confidence interval of a control population (11 men, 9 women; $20-77$ years old; mean age: 52 years). ARs and SRs of both populations are derived from the same type of speech material, which is described in the section 'Text Passages'. The mean SR of the control group was 3.36 syllables/s $(\mathrm{SE}=0.12)$. The corresponding prediction interval ranged from 2.3 to 4.5 syllables/s. The mean AR was 4.70 syllables/s $(\mathrm{SE}=0.13)$ with the $95 \%$ prediction interval ranging from 3.5 to 5.9 syllables/s.

\section{Rate Control Methods}

Seven RCMs were selected. Speaking slower on demand was the only flexible method. The participants were instructed to speak half as fast as they were used to. Rigid methods used were: alphabet board, hand tapping, pacing board, delayed auditory 
feedback with a delay of $50 \mathrm{~ms}$ (DAF50), delayed auditory feedback with a delay of $100 \mathrm{~ms}$ (DAF100) and delayed auditory feedback with a delay of $150 \mathrm{~ms}$ (DAF150).

\section{Text Passages}

Many dysarthric speakers demonstrate difficulties with production of spontaneous speech. Therefore, reading samples were preferred above spontaneous speech samples. Furthermore, all published data concerning the effect of speech rate on intelligibility are based on reading passages.

For this task, 20 different passages with simple sentence constructions and without any difficult word choices were selected. For each method, the participants were asked to read a randomly selected reading passage for at least $2 \mathrm{~min}$. To be sure that the results would not be influenced by the reading level of the judged paragraphs, the exact reading level had been calculated, applying the reading index of Brouwer [15]. By means of this systems, text fragments can be differentiated into 9 reading levels. The reading level of a text is based on the mean number of words per sentence and the mean number of syllables per word. All selected reading passages had reading level 7 (mean sentence length: 9 words; mean word length: 1.34-1.39 syllables) or 8 (mean sentence length: 10 words; mean word length: $1.38-1.44$ syllables). The differences between both reading levels can be considered as small. Additionally, the differences between the mean reading levels of all RCMs are also very small.

\section{Speech Samples}

Two-minute samples were recorded for each of the eight assessed conditions: habitual SR and the seven RCMs. Each subject started with habitual SR. For the RCMs a randomized sequence was used. The speech samples were recorded in a quiet environment by means of a notebook (HP Pavilion ze4600) and a free available wave editor (Audacity ${ }^{\circledR}$ ). The mouth-to-microphone (Sony ECM-717) distance measured $300 \mathrm{~mm}$. The digital speech samples had a sampling frequency of $44 \mathrm{kHz}$. Each sample was saved as a separate wave file.

\section{Intelligibility Ratings}

The intelligibility of the speech samples was rated by 5 speechlanguage pathologists with experience in dysarthria. They were not familiar with the reading passages. According to a previously used definition of intelligibility $[15,16]$, the listeners were instructed to indicate the degree to which they understood the utterances produced by the speaker on a $100-\mathrm{mm}$ visual analogue scale (VAS). The listeners were asked to exclude perceptual judgments of naturalness, voice quality, pitch and so on from the intelligibility rating. The extremities of the VAS were respectively labeled as completely unintelligible and completely intelligible. Listeners reported normal hearing and were native speakers of Dutch.

The 152 samples (19 participants $\times 8$ conditions) were randomly played back by means of Windows Media Player, installed on a notebook (HP pavilion ze4600). As the samples were judged by all the listeners simultaneously, speakers (Logitech) were used to present the samples at a SPL of $75-80 \mathrm{~dB}$.

\section{Inter-Rater Reliability for the Intelligibility Ratings}

Inter-rater reliability for the intelligibility ratings was determined for all 152 samples (19 dysarthric speakers $\times 8$ methods) by means of an intraclass correlation coefficient. A strong inter-
Table 2. Habitual and modified ARs and SRs of the dysarthric population

\begin{tabular}{llcl}
\hline Method & SR, syllables/s & AR, syllables/s & $\mathrm{n}$ \\
\hline Habitual & $2.75 \pm 0.15$ & $4.79 \pm 0.36$ & 19 \\
Voluntary & $2.51 \pm 0.17$ & $4.7 \pm 0.4$ & 19 \\
Alphabet board & $0.60 \pm 0.06$ & $3.2 \pm 0.4$ & 18 \\
Hand tapping & $1.54 \pm 0.12$ & $3.23 \pm 0.24$ & 19 \\
Pacing board & $1.02 \pm 0.08$ & $2.89 \pm 0.27$ & 18 \\
DAF50 & $2.46 \pm 0.16$ & $3.83 \pm 0.29$ & 19 \\
DAF100 & $2.22 \pm 0.16$ & $3.33 \pm 0.25$ & 19 \\
DAF150 & $1.99 \pm 0.18$ & $3.12 \pm 0.3$ & 19 \\
\hline
\end{tabular}

Figures are means $\pm \mathrm{SE}$.

rater reliability was found (intraclass correlation coefficient: $0.854)$. The mean error rate was $8 \%$.

\section{$S R$ and $A R$}

For each sample, SR (syllables per second, pauses included) and AR (syllables per second, pauses excluded) were calculated. The calculations are based on 1-min mid-sample segments to avoid potential passage beginning and ending effects. The number of perceived syllables produced during that minute, including filled pauses, was counted. To calculate AR all silent pauses were eliminated. Silent pauses were defined as a silent fragment of at least $200 \mathrm{~ms}$ [17].

\section{Statistical Analyses}

Statistical analyses were performed by means of SPSS 14 . The effect of RCMs on SR, AR and intelligibility was examined by means of repeated-measures ANOVA. For the post hoc pairwise comparisons the Bonferroni correction was applied. Significance level was set at $\mathrm{p}<0.05$.

\section{Results}

\section{Effects of Rate Control on SR}

Table 2 shows the mean SRs of the dysarthric group for the seven examined speech RCMs. A significant effect of methods was found ( $\mathrm{p}<0.001$ ). Pairwise comparison showed that each RCM resulted in significantly lower mean SRs compared with the habitual rate $(\mathrm{p}<0.05$; $\mathrm{p}<$ 0.001 for alphabet board, hand tapping and pacing board; fig. 1). The impact of rate control on SR varied between methods. The differences between RCMs were also found to be significant ( $\mathrm{p} \leq 0.001 ; \mathrm{p}<0.05$ for hand tapping vs. DAF100), with the exception of the following differences: voluntary vs. DAF50, voluntary vs. DAF100, voluntary vs. DAF150, hand tapping vs. DAF150, DAF50 vs. DAF100 and DAF50 vs. DAF150. 
Fig. 1. Habitual and modified ARs and SRs of the dysarthric population.

Fig. 2. Effect of RCMs on the intelligibility of dysarthric speakers.
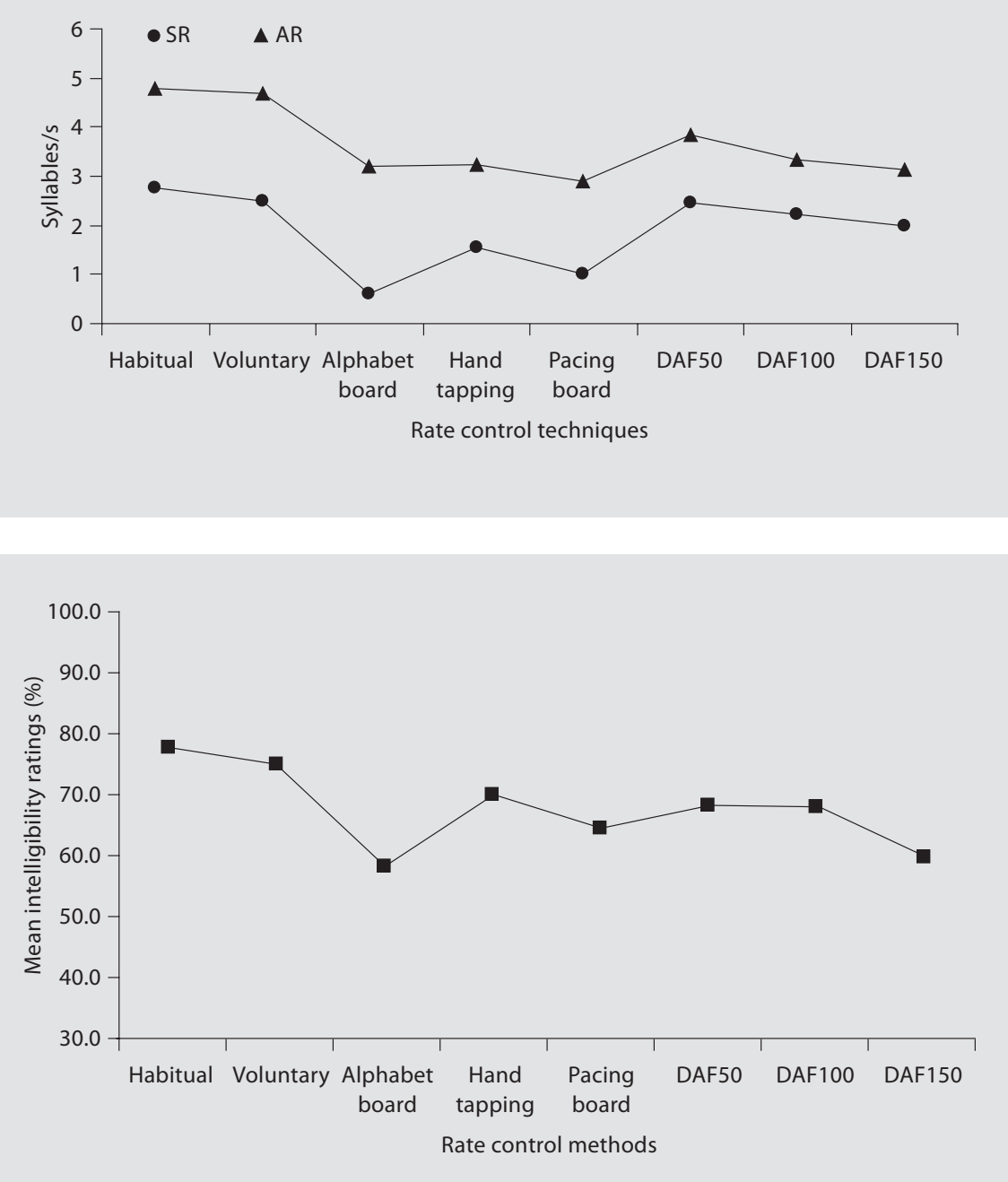

\section{Effects of Rate Control on $A R$}

Table 2 presents the mean ARs of the dysarthric group for the seven examined speech RCMs. The effect of methods was found to be significant $(\mathrm{p}<0.001)$. Post hoc pairwise comparison showed that each RCM resulted in significantly lower ARs when compared to the habitual AR $(p<0.001)$, with the exception of voluntary rate control (speaking slower on demand) (fig. 1). The differences between AR for voluntary rate control and the other methods were all significant ( $\mathrm{p} \leq 0.001 ; \mathrm{p}<0.05$ for voluntary rate control and DAF50). Additionally, a significant difference was found between AR DAF50 and AR pacing board $(\mathrm{p}<0.001)$.

\section{Speech Rate and Intelligibility}

Figure 2 illustrates the effect of the investigated RCMs on the mean intelligibility rating of the dysarthric group. The VAS ranges from 0 (completely unintelligible) to 100 (completely intelligible). The effect of methods was found to be significant $(\mathrm{p}<0.001)$. However, none of the RCMs resulted in a higher mean intelligibility rating. The mean intelligibility ratings for DAF100 and DAF150 even differed significantly $(p<0.05)$ from the mean intelligibility rating of the habitual speaking mode. However, when looking at individual results (table 3), all RCMs resulted in improved intelligibility in 1 or more dysarthric speakers. In 12 of the 19 dysarthric speakers, intelligibility improved by at least $1 \%$. However, the increased intelligibility ratings should be interpreted with- 
Table 3. Effect of RCMs on the dysarthric speakers' intelligibility

\begin{tabular}{|c|c|c|c|c|c|c|c|c|}
\hline \multirow{2}{*}{$\begin{array}{l}\text { Method } \\
\text { subject }\end{array}$} & \multicolumn{8}{|c|}{ Speech intelligibility ratings, \% } \\
\hline & habitual & voluntary & $\begin{array}{l}\text { alphabet } \\
\text { board }\end{array}$ & $\begin{array}{l}\text { hand } \\
\text { tapping }\end{array}$ & $\begin{array}{l}\text { pacing } \\
\text { board }\end{array}$ & DAF50 & DAF100 & DAF150 \\
\hline 1 & 82.8 & 84.4 & 77.8 & $88.6^{\mathrm{b}}$ & 75.4 & 79.2 & 75.2 & 54.8 \\
\hline 2 & 90.6 & $94.4^{\mathrm{a}}$ & 64.6 & 83.2 & 71.4 & 75.2 & 66.8 & 67.2 \\
\hline 3 & 95.2 & 89 & 77 & 79.6 & 82 & 88 & 86.8 & 85.2 \\
\hline 4 & 86.2 & $89.8^{\mathrm{a}}$ & 84 & 83.8 & $88.4^{\mathrm{a}}$ & 70 & 76.8 & 81.2 \\
\hline 5 & 79.6 & $82.4^{\mathrm{a}}$ & 53 & 68.6 & 35.8 & 64.2 & 68.4 & 53.8 \\
\hline 6 & 78.4 & $90.6^{c}$ & $92^{\mathrm{c}}$ & $83^{\mathrm{a}}$ & $81.2^{\mathrm{a}}$ & 74.2 & $81.6^{\mathrm{a}}$ & 59.2 \\
\hline 7 & 94.2 & 94.4 & 78 & 92.2 & 79.8 & 89 & 86.2 & 91.2 \\
\hline 8 & 58.4 & $62^{\mathrm{a}}$ & 19 & 52.2 & 46.4 & $61.4^{\mathrm{a}}$ & $63.8^{\mathrm{b}}$ & 48 \\
\hline 9 & 67.2 & 51.6 & 4.4 & 1.8 & 0.6 & 15.6 & 55.6 & 7 \\
\hline 10 & 97.4 & 90 & 85.2 & 87.8 & 84.8 & 92.8 & 90.8 & 88.4 \\
\hline 11 & 37.6 & 11.8 & 9 & 30.8 & 1 & 1.2 & 15.6 & 12.6 \\
\hline 12 & 89.2 & 79.8 & 86.6 & 75 & 84.2 & 78.4 & 75.4 & 82.6 \\
\hline 13 & 48.6 & $57^{\mathrm{c}}$ & 11 & $56^{\mathrm{b}}$ & $59.2^{\mathrm{c}}$ & $50.6^{\mathrm{a}}$ & 39.4 & 32.6 \\
\hline 14 & 94.8 & 93.2 & 58 & 92.8 & 84.6 & 90.6 & 81.2 & 66.6 \\
\hline 15 & 88.4 & $95^{\mathrm{b}}$ & 33.6 & 77.6 & 59.8 & 85.8 & 83.2 & 65.2 \\
\hline 16 & 46 & 45 & $77.6^{\mathrm{c}}$ & $57.8^{\mathrm{c}}$ & $59^{c}$ & 41.4 & 28.2 & 37.6 \\
\hline 17 & 83.6 & 69.4 & 79 & $92.4^{c}$ & 78.2 & 84 & 66.4 & 59.8 \\
\hline 18 & 80.6 & 70 & 59.8 & 54.2 & $88.8^{\mathrm{c}}$ & $87.8^{\mathrm{b}}$ & $83.2^{\mathrm{a}}$ & 81.4 \\
\hline 19 & 15.8 & $18.8^{\mathrm{a}}$ & - & 5.8 & - & 11.6 & 44 & 2.6 \\
\hline
\end{tabular}

${ }^{\mathrm{a}}$ Intelligibility improved by $1-5 \% .{ }^{\mathrm{b}}$ Intelligibility improved by $5-8 \% .{ }^{\mathrm{c}}$ Intelligibility improved by more than $8 \%$.

in the framework of the reliability data. The mean percentage error for the intelligibility ratings indicated that changes in intelligibility of less than $8 \%$ cannot be completely ascribed to rate control. Thus, only changes of more than $8 \%$ are meaningful. Such an increase only occurred in 5 speakers (subjects $6,13,16,17$ and 18). Note that intelligibility improved between 5 and $8 \%$ in 3 additional subjects (subjects 1, 8 and 15). Table 4 summarizes the characteristics of the 5 speakers for whom an increase of intelligibility of at least $8 \%$ secondary to one or more RCMs was found. It shows that most subjects' habitual articulation and SRs fitted within the 95\% confidence interval of the control group. Additionally, the effect of rate control seemed not to be related to a specific type of dysarthria. Also note that the degree of habitual intelligibility of the 5 dysarthric speakers varied from 46 to $83.6 \%$. The RCMs responsible for an increase of at least $8 \%$ were: voluntary rate control $(\mathrm{n}=2)$, alphabet board $(\mathrm{n}=2)$, hand tapping $(\mathrm{n}=2)$, and pacing board $(\mathrm{n}=3)$.

Table 3 also shows that rate control may have an inverse effect on intelligibility. A decrease of intelligibility of more than $8 \%$ occurred in each participant, independently of the applied RCM.

Rate Control and Intelligibility
Table 4. Characteristics of speakers with an increase of intelligibility of at least $8 \%$ secondary to rate control

\begin{tabular}{rlllll}
\hline $\begin{array}{l}\text { Sub- } \\
\text { ject }\end{array}$ & Subtype & $\begin{array}{l}\text { Speaking } \\
\text { rate }\end{array}$ & $\begin{array}{l}\text { Articulation } \\
\text { rate }\end{array}$ & $\begin{array}{l}\text { Habitual } \\
\text { Intelligi- } \\
\text { bility, \% }\end{array}$ & $\begin{array}{l}\text { Maximal in- } \\
\text { crease intel- } \\
\text { ligibility, \% }\end{array}$ \\
\hline 17 & Hypokinetic & normal & normal & 83.6 & 8.8 \\
18 & Hypokinetic & normal & increased & 80.6 & 8.2 \\
6 & Ataxic & normal & normal & 78.4 & 13.6 \\
13 & UUMND & normal & normal & 48.6 & 10.6 \\
16 & UUMND & normal & decreased & 46 & 31.6 \\
\hline
\end{tabular}

UUMND = Unilateral upper motor neuron dysarthria.

\section{Discussion}

This study investigated the effect of several RCMs on AR, SR and intelligibility. The results reveal that all RCMs result in slower ARs and SRs, with the exception of AR for voluntary rate control. Rate control did not improve the overall intelligibility of the dysarthric population. However, a meaningful increase of more than $8 \%$ was registered in 5 speakers.

Folia Phoniatr Logop 2009;61:69-75 


\section{Rate Control and Speech Rate}

This study confirms that the RCMs used in clinical practice to reduce speech rate are effective. All investigated methods result in significantly lower mean SRs and, with the exception of voluntary rate control, also in significantly lower ARs. Pacing board, hand tapping, alphabet board and DAF150 have the highest impact on both SR and AR.

\section{Effect of Rate Control on Intelligibility}

The results of previous studies concerning the effect of rate control on intelligibility are rather heterogeneous. Some studies revealed a positive effect of reduced speech rates on intelligibility in dysarthric speakers [7-9, 12-14], whereas other studies did not find such an effect [5, 9-11]. In the currently investigated dysarthric population, reduced SRs and ARs did not result in higher mean intelligibility ratings. Lower speech rates even resulted in lower levels of intelligibility. Table 3 shows that decreased intelligibility ratings were found in each subject. A decrease of more than $8 \%$ - the mean percentage error for the intelligibility ratings was $8 \%$ - even occurred in each of the 19 participants, independently of the applied RCMs. This is in great contrast with the expectations and presumptions based on the existing literature $[1,2,5-9]$. In case of a pacing board, an alphabet board and hand tapping, this effect may be attributed to the fact that these methods require a certain degree of attention and coordination. The influence of these aspects may be high since the subjects were not familiar with the methods. The same may be true for delayed auditory feedback $[16$, 18].

In contrast, intelligibility increased with at least $1 \%$ secondary to one or more RCMs in 12 of the 19 dysarthric speakers. However, taking into account the mean percentage error for intelligibility, only increases of more than $8 \%$ can be judged as meaningful. Such increases occurred in only 5 subjects. The fact that the habitual ARs and SRs of most of these speakers fit within normal boundaries supports the assumption that rate control is not only useful for dysarthric speakers with increased speech rates $[1,4,12]$. Thus, rate control should be considered as a possible aspect of speech therapy for every dysarthric speaker, independently of the speaker's habitual speech rate. Improvement of intelligibility secondary to rate control has mainly been described in persons with hypokinetic or ataxic dysarthria [8, 9, 12-14]. This study confirms that persons with hypokinetic and ataxic dysarthria may benefit from rate control. Additionally, rate control can also be useful in persons with unilateral up- per motor neuron dysarthria. Not all subjects, though, with hypokinetic, ataxic or unilateral upper motor neuron dysarthria who participated in this study had increased intelligibility ratings secondary to rate control. It seems that the effect of rate control cannot be related to a specific type of dysarthria.

Another important issue is the correlation between the RCM and the impact on intelligibility. The methods responsible for a meaningful increase of intelligibility are: voluntary rate control, pacing board, alphabet board and hand tapping. In 8 of the 19 participants, voluntary rate control caused an increase of intelligibility of more than $2.8 \%$. This is rather unexpected since the effect of voluntary rate control on AR was insignificant and the impact on SR was smaller than the impact of other methods. However, voluntary rate control is the only flexible RCM. Although the patient is instructed to speak slower, the pauses will still be more weighted and natural than when using rigid RCMs. This might have an important impact on a person's intelligibility. On the other hand, this study uses intelligibility ratings rather than percentages of words correctly understood. The listeners were instructed to judge intelligibility, but ratings could have been influenced by the perceived naturalness of the utterance. Transcribing the utterances might provide additional insights. Although the alphabet board increased intelligibility in 2 participants by more than $8 \%$, it also decreased intelligibility in 12 subjects by more than $8 \%$. A similar effect was found for the use of a pacing board, hand tapping and to a lesser extent for voluntary rate control. DAF150 even resulted in decreased intelligibility in 18 of the 19 subjects. In 15 cases, the decrease was more than $8 \%$.

Another important question is whether clinicians should rely on the degree of intelligibility to decide whether or not a dysarthric patient should get rate control therapy. The intelligibility ratings of the 5 speakers with a significant increase ranged from 46 to $83.6 \%$, indicating that overall intelligibility does not need to be severely decreased to benefit from rate control. Note, however, that an improvement of more than $5 \%$ did not occur in persons with an intelligibility level higher than $90 \%$. Furthermore, 2 of the 3 subjects who experienced an increase of more than $10 \%$ had intelligibility ratings below $50 \%$. This suggests that the effect of rate control may be higher in patients with severely decreased intelligibility.

The comparison of several RCMs is probably the most important additional value provided by this study. All RCMs were found to reduce AR and SR to a significant degree - with the exception of voluntary rate control for 
AR. Regarding improvement of intelligibility, voluntary rate control, pacing board, alphabet board and hand tapping were found to be the most effective methods. Rate control may be effective for different types of dysarthria and not only for hypokinetic and ataxic dysarthria. Finally, a patient's intelligibility must not be profoundly decreased to benefit from rate control, but degree of intelligibility may be an influencing factor.

Based on the current results, we were not able to compose a profile of patients who may benefit from a certain rate control strategy. Further investigation with a larger population and a greater range of severity seems to be necessary.

Although the results of the current study and the results available in the literature are heterogeneous, rate control should not be omitted as a part of speech therapy in patients with dysarthria, since some patients do benefit from rate reduction. It is important that clinicians find out which effect a specific RCM has on a specific patient.

\section{Limitations of the Study}

The authors are well aware of three disadvantages of the methods. Firstly, since the dysarthric population was heterogeneous, the current study does not allow to draw conclusions for a certain type of dysarthria. Secondly, most speakers had only mildly to moderately decreased running speech intelligibility. Finally, since the current study uses intelligibility ratings instead of measurements, one should be careful when comparing the results of this study with those of previous studies.

\section{Acknowledgments}

This work was supported by the Flemish Institute for the Promotion of Innovation by Science and Technology in Flanders (IWT, contract SBO/40102). The authors also acknowledge their colleagues Nele Wallyn and Sarah Van Der Donck who assisted in the data collection.

\section{References}

1 Marshall RC, Karow CM: Retrospective examination of failed rate-control intervention. Am J Speech Lang Pathol 2002;11:316.

2 Yorkston KM, Dowden PA, Beukelman D: Intelligibility as a tool in the clinical management of dysarthric speakers; in Kent RD (ed): Intelligibility in Speech Disorders: Theory, Measurement and Management. Amsterdam, Benjamin, 1992, pp 265-286.

$\checkmark 3$ Jaeger M, Hertrich I, Stattrop U, Schonle PW, Ackermann H: Speech disorders following severe traumatic brain injury: kinematic analysis of syllable repetitions using electromagnetic articulography. Folia Phoniatr Logop 2000;52:187-196.

$\checkmark 4$ McHenry MA: The effect of pacing strategies on the variability of speech movement sequences in dysarthria. J Speech Lang Hear Res 2003;46:702-710.

$\checkmark 5$ Tjaden K, Wilding GE: Rate and loudness manipulations in dysarthria: acoustic and perceptual findings. J Speech Lang Hear Res 2004;47:766-783.

6 Caligiuri MP: The influence of speaking rate on articulatory hypokinesia in parkinsonian dysarthria. Brain Lang 1989;36:493-502.
7 Turner GS, Tjaden K, Weismer G: The influence of speaking rate on vowel space and speech intelligibility for individuals with amyotrophic lateral sclerosis. J Speech Hear Res 1995;38:1001-1013.

8 Yorkston KM, Beukelman DR, Strand EA, Bell KR: Management of Motor Speech Disorders in Children and Adults. Austin, ProEd, 1999.

9 Hammen VL, Yorkston KM, Minifie FD: Effects of temporal alterations on speech intelligibility in parkinsonian dysarthria. J Speech Hear Res 1994;37:244-253.

10 Dagenais PA, Brown GR, Moore RE: Speech rate effects upon intelligibility and acceptability of dysarthric speech. Clin Linguist Phon 2006;20:141-148.

11 Weismer G, Laures JS, Jeng JY, Kent RD, Kent JF: Effect of speaking rate manipulations on acoustic and perceptual aspects of the dysarthria in amyotrophic lateral sclerosis. Folia Phoniatr Logop 2000;52:201-219.

12 Yorkston KM, Hammen VL, Beukelman DR, Traynor CD: The effect of rate control on the intelligibility and naturalness of dysarthric speech. J Speech Hear Disord 1990;55:550560 .
13 Downie AW, Low JM, Lindsay DD: Speech disorders in parkinsonism: usefulness of delayed auditory feedback in selected cases. $\mathrm{Br}$ J Disord Commun 1981;16:135-139.

14 Hanson W, Metter E: DAF speech rate modification in Parkinson's disease: a report of two cases; in Berry W (ed): Clinical Dysarthria. Austin, Pro-Ed, 1983, pp 231-254.

15 Visser J, van Laarhoven A, ter Beek A: AVIToetspakket Handleiding. 's Hertogenbosch, KPC, 1994.

16 Rousseau B, Watts CR: Susceptibility of speakers with Parkinson disease to delayed feedback. J Med Speech Lang Pathol 2002;10: 41-49.

17 Turner GS, Weismer G: Characteristics of speaking rate in the dysarthria associated with amyotrophic lateral sclerosis. J Speech Hear Res 1993;36:1134-1145.

18 Dagenais PA, Southwood MH, Lee TL: Rate reduction methods for improving speech intelligibility of dysarthric speakers with Parkinson's disease. J Med Speech Lang Pathol 1998;6:143-157. 